\title{
On the dynamic nature of the prolate solar chromosphere: jet formation
}

\author{
B. Filippov ${ }^{\star}$, S. Koutchmy, and J. Vilinga
}

\begin{abstract}
Institut d'Astrophysique de Paris, CNRS and Univ. P. \& M. Curie, 98bis boulevard Arago, 75014 Paris, France e-mail: bfilip@izmiran.troitsk.ru; [koutchmy;vilinga]@iap.fr
\end{abstract}

Received 1 September 2006 / Accepted 24 October 2006

\begin{abstract}
Context. In "cool" spectral lines, the smoothed upper edge of the solar chromosphere is prolate in the South-North direction at the epoch of minimum solar activity and nearly spherically symmetric at the maximum phase. We attribute the effect to the dynamical nature of the upper chromosphere, which consists of a large number of small jet-like structures ascending into the corona. We could not resolve the source region of an individual jetlet, although similar but larger structures are visible, especially in EUV coronal lines. Aims. We consider the problem of the formation of an individual jet above the limb, assuming that a large number of jet-like events is responsible for the prolate solar upper chromosphere. We then assume that spicules, being the cool part of the phenomenon, behave similarly, and we will mainly concentrate the analysis on the magnetic origin of the event.

Methods. Image processing is used to reveal the displacement of magnetic field tubes filled with coronal plasma and jet formation due to field aligned motion above the null point created in the corona by the emerging magnetic bipole.

Results. The growth of the bipole leads to a reconnection of the field lines and to a specific plasma motion in the vicinity of the null point that results in a plasma flow along the spine line of the 3D null. We assume that similar but smaller processes could happen very often at a smaller scale in the chromosphere, near emerging magnetic ephemeral regions, forming numerous jetlets in the upper chromosphere. As the field aligned motion is guided by the magnetic field, at the epoch of low activity the large-scale structure of the polar magnetic field and the one of the quiet equatorial region is sufficiently different to explain the prolateness of the chromosphere.
\end{abstract}

Key words. Sun: chromosphere - Sun: corona - Sun: magnetic fields

\section{Introduction}

The solar chromosphere above $2 \mathrm{Mm}$ is not a perfect spherically stratified atmosphere as assumed in classical hydrostatic and/or in 1D models. The upper edge of the chromosphere seen at moderate resolution in strong chromospheric emission lines is rather "blurred" as it consists of the mixture of a large number of jet-like dynamic spicules and of coronal plasma between them (Fig. 1). Each spicule has a lifetime of no more than 20 min during which it elongates, reaches its highest height, and then falls down (Sterling 2000). The superposition of a host of spicules along a line of sight forms the visual upper edge of the chromosphere. Further we will use the word spicule to designate the cool part (made of low ionization plasma) of a jet-like phenomenon occurring at chromospheric heights $(2$ to $10 \mathrm{Mm})$. Spicules are usually seen all around the limb of the Sun; they are not confined to the area occupied by coronal holes, but they should not be confused with the fibrils of lower altitudes, see Athay (1986).

Many past observations showed that at the epoch of solar minimum the extension of the chomosphere near the poles is systematically higher than at the equator (Secchi 1877; Roberts 1946; Fracastoro 1948; Athay 1959; Dunn 1960) and modern precise measurements confirmed and substantiated these early suggestions (Johannesson \& Zirin 1996; Auchere et al. 1998; Zhang et al. 1980; Georgakilas et al. 1999; Vilinga \& Koutchmy 2005). The amount of prolateness depends on the amount of

* Permanent address: Pushkov Institute of Terrestrial Magnetism, Ionosphere and Radio Wave Propagation, Russian Academy of Sciences, Troitsk Moscow Region, 142190, Russia. plasma that emits at the observed light. In the transition region (TR) resonance line of He II at $30.4 \mathrm{~nm}$, the effect seems more prominent than in a chromospheric cool Balmer line like $\mathrm{H} \alpha$ of $\mathrm{HI}(\sim 8000 \mathrm{~K})$ because additional structures appear at TR temperatures higher up, especially over coronal holes $(\mathrm{CH})$. In the $\mathrm{H} \alpha$ line of $\mathrm{HI}$, the prolateness is greater than in the Ca II $\mathrm{K}$ line $(\sim 6500 \mathrm{~K})$. In hot coronal lines, the prolateness is not observed in emission; indeed the chromosphere appears in absorption as a dark band that extends from $5 \mathrm{Mm}$ up to $9 \mathrm{Mm}$ and that has been resolved only recently (see Daw et al. 1995). In the HeI lines, which are mainly formed low in the chromosphere (typically at a $1.5 \mathrm{Mm}$ height), the prolateness effect cannot be measured, partly because these lines show a "reversed" $\mathrm{CH}$ effect. Again, the prolateness effect should not be confused with the CH effect (see Koutchmy \& Loucif 1991; Filippov \& Koutchmy 2000; Vilinga \& Koutchmy 2005), which is revealed at higher TR temperatures where macrospicules are observed and which is less extended in latitude.

The prolateness effect depends on the phase of the solar activity cycle. It was very clearly visible at the epoch of solar minimum, but it disappeared near the maximum (Vilinga \& Koutchmy 2005), although this variation has to be measured more carefully. At activity decay phase, the prolateness appeared again, but there is no detailed study of the effect through the cycle. The solar cycle dependence of the prolateness suggests that the magnetic field should play an important role in its formation. In a previous paper (Filippov \& Koutchmy 2000), we proposed a simple geometrical model of the influence of the magnetic field structure on the average height of the dynamic 


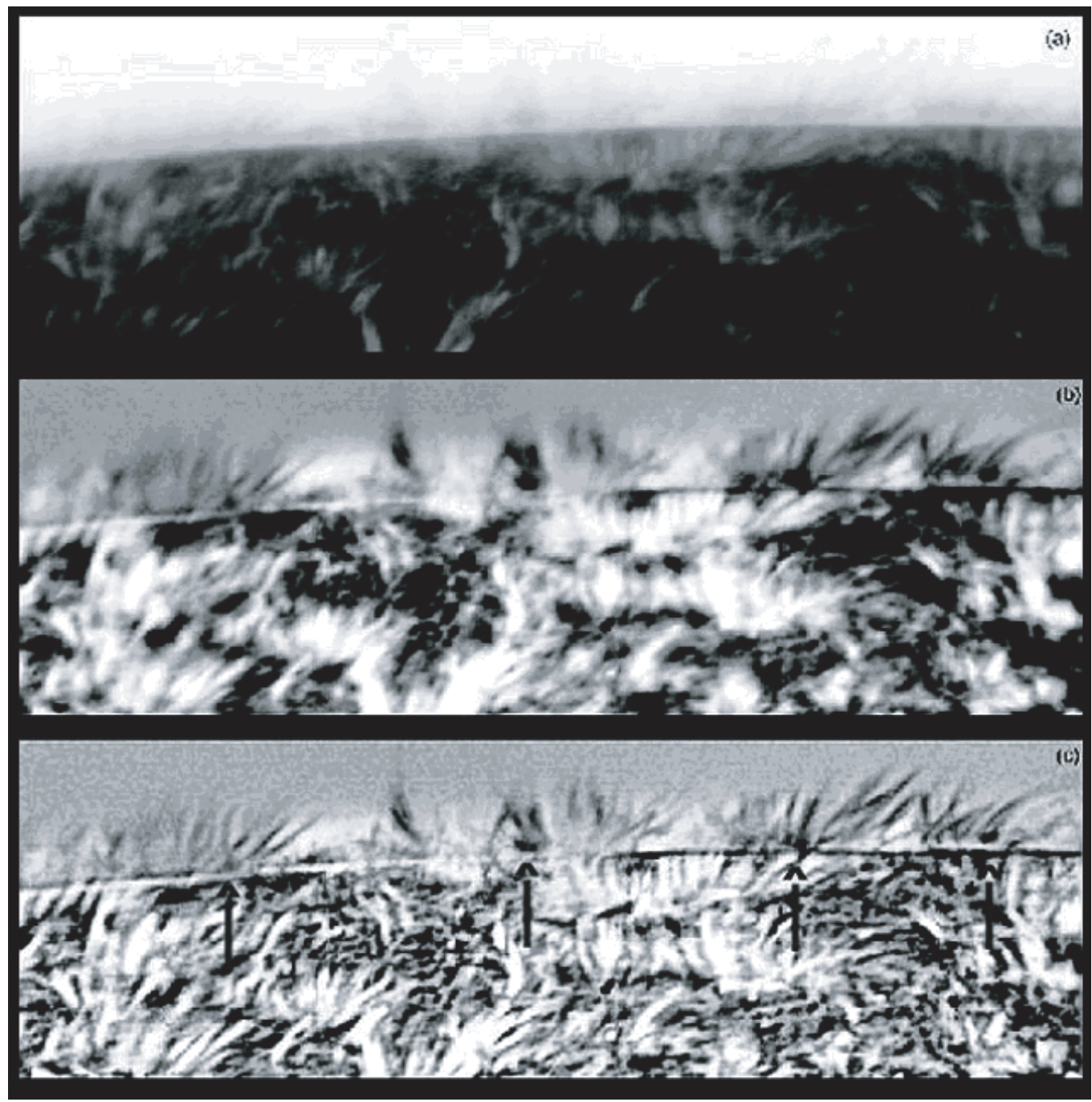

Fig. 1. Jet-like structures in the upper polar region chromosphere, as seen in negative, from observations taken in the blue wing of the H $\alpha$ line, before a) and after b) image processing to remove the large background variations, and after c) using the unsharp mask (from Christopoulou et al. 2001; observations from the VTT RBDunn at NSO/SacPeak, using the UBF filter). Black arrows inserted in c) show examples of spicules with possible small loops or arch-shaped mottles at their feet.

chromosphere, which nevertheless quantitatively explained the prolateness effect and its dependence on the temperature of the observed plasma. The difference in the height of polar and equatorial chromospheres arises due to the difference in structure of polar and low latitude magnetic fields. It is well-known that the large-scale magnetic field in the polar region is mostly open at the sunspot minimum, while in the equatorial region it is mostly closed according to the dominance of the global dipolar and octupolar spherical harmonics. The small-scale structure of the magnetic field in the two regions is likely not be the same. The real fine structure of the polar magnetic field is elusive. There are strong suspicions that it is very similar to the fine structure of the magnetic field within coronal holes sometimes observed at low latitudes. A detailed analysis of high-resolution magnetograms shows that network magnetic elements within the coronal holes are predominately of the same polarity. The ratio of areas occupied by opposite polarities is 1:4 within the coronal holes, while it is about 1:1 in quiet regions (Varsik et al. 1999; Belenko 2001). In regions of mixed polarities, a lot of field lines do not rise high into the corona as they connect the nearby patches of opposite polarity, whereas in the regions of predominant polarity most of the field lines reach high altitude (Fig. 2).
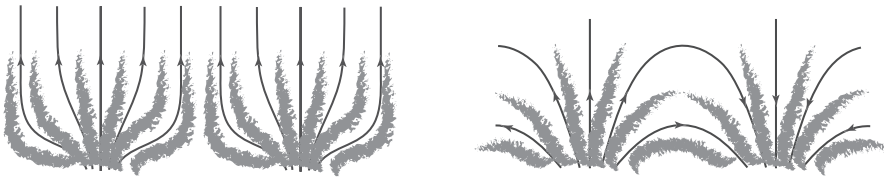

Fig. 2. Schematic representation (not to scale) of the magnetic field lines and plasma jets at the pole (left) and at the equator (right).

Magnetic fields usually guide the mass flows along the field lines. For a ballistic jet motion (which is not exact for a large part of spicules, but can be assumed for the purpose of this analysis), the maximum height of a spicule would be the same for all trajectories along the curved field lines if a line reaches this height. In polar regions, all cool jets are able to ascend up to a gravitationally limited height. The mean density distribution of the cool gas in the upper polar chromosphere is determined by the "dynamic" (not hydrostatic) scale height in an individual spicule and the distribution of the number of spicules versus the height. In the equatorial region, some jets will be forced to come down after they reach the apex of a relatively low arch. When considering the time averaged heights reached by spicules, this 
factor will reduce the mean chromospheric density at a given height, proportionally to the decrease of the magnetic flux of one polarity.

We attempted to calculate the magnetic field using direct measurements of the field in the photosphere and to find out the difference in the magnetic field scales in the equatorial and in the polar regions (Filippov et al. 2002). Contrary to the schematic representation shown in Fig. 2, magnetic field calculations showed that the magnetic flux decreased with height both in the equatorial region and in the polar region. In fact, the situation is intermediate between the cases shown in the left-hand and right-hand parts of Fig. 2. The net magnetic flux through a given region of the photosphere is non-zero, and it is not composed of one single polarity surface element. However, we may expect the decrease of the flux of one polarity in the polar region to be slower. Calculations show that the scale of the magnetic field in the polar region is really greater than the field scale in the equatorial region, but magnetic field data in the regions so close to the polar limbs are too inaccurate and noisy to make a reliable quantitative definite conclusion. The statistical effect of the polar field is then difficult to predict in the high chromosphere, although it is evidently greatly determined by the magnetic fluxes observed at the photospheric level and their small-scale motions.

In this paper, we will consider the formation of an individual jet above the limb, assuming that a large number of jet-like events is responsible for the prolate solar upper chromosphere. We then assume that spicules, being the cool parts of the phenomenon, behave similarly, and we will mainly concentrate the analysis on the magnetic origin of the event.

\section{Analysis of the small jet formation}

A wide range of various jet-like structures is observed in the solar atmosphere. They can be formed both from i) cool plasma as spicules and surges, and ii) hot plasma like X-ray coronal jets (Beckers 1972; Rompolt \& Svestka 1996; Shibata et al. 1992; Koutchmy et al. 1997; Koutchmy et al. 1998; Wang et al. 1998; Chae et al. 1999). Accordingly, we define a spicule as the cool counterpart of the jet phenomenon, which involves a small magnetic eruption possibly with a coronal core. For a more comprehensive review of the jet/spicule phenomena, see the relevant papers on jets and spicules in the Proceedings of the Guadeloupe Conference (1998) published as an ESA SP-421.

The length of jets varies from a few megameters for spicules up to hundreds of Mm for X-ray jets. No doubt all of them are collimated and guided by magnetic fields. Although the mechanism of jet formation is not yet clear, many models try to ascribe a more active role to the magnetic field than just that of guiding the mass flow. In the more extreme cases, $\boldsymbol{J} \times \boldsymbol{B}$ force or electric fields were suggested to accelerate the plasma flows (see Shibata \& Uchida 1986; Lorrain \& Koutchmy 1996; Henoux \& Somov 1997). Schluter (1957) proposed a "melon-seed" mechanism for diamagnetic plasma to be forced out of the diverging magnetic field. Platov et al. (1973) calculated the raking-up of plasma due to the growth of the local magnetic field. For a surge formation in $\sim 1$ min, the magnetic moment of a small magnetic concentration (local bipole) should grow by a factor of $\sim 25$. Filippov (1993) also considered surge formation by the compression of the lower part of a vertical magnetic tube due to the growth of the local magnetic field. A system of hydrodynamic equations for an ideal gas in a flux tube was solved numerically. An increase in the field strength with a timescale of less than $300 \mathrm{~s}$ is necessary for the efficient formation of a jet having the characteristics of a typical surge. Special attention is paid to spicular models in which field line reconnection ${ }^{1}$ took place, starting with Pikel'ner (1969), Uchida (1969), Blake \& Sturrock (1985), and going to the more recent numerical simulations by Shibata et al. (1994), Karpen et al. (1995), Yokoyama \& Shibata (1996), and Karpen et al. (1998).

We note that many jets are associated with emerging small flux regions that manifest also themselves as brightenings in chromospheric and coronal spectral lines. Such a new patch of parasitic polarity within a large-scale unipolar area inevitably leads to the appearance of a new null point (X-point) in the chromosphere or corona. However, it will just be a single 3D null point (Filippov 1999), not a line, so it is difficult to imagine the development of any current sheet associated with it. The situation is quite different with the case of a two-ribbon flare where the 2D approximation seems reasonable because the dimension along the neutral line has a scale significantly exceeding the scales in the other two dimensions. Accordingly, even the naive approach that we assume to simply describe the theoretical initial conditions leads to a problem. It is then important to come back to the observations to find some guidance.

To perform our analysis, we selected a clear observation of a jet formation above an X-point in the corona. The event was observed by the Transition Region and Coronal Explorer (TRACE) camera on 3 October 2001 (Fig. 3). A movie of the event can be found at the TRACE Web site in the collection of movies as the movie: \#50 from (http://vestige.lmsal.com/TRACE/Public/Gallery/ Images/TRACEpod.html).

The structure is similar to the saddle structure in AR 8113 on December 1997 shown in Fig. 2 of Filippov (1999), although the scale of the formation is now about a factor of 5 smaller! Another distinction in the two patterns is in the configuration of the external magnetic field. The December 1997 saddle was formed near the outer boundary of the arcade of active region loops, while the 3 October 2001 saddle was located within a unipolar region. There were no prominent jets observed in AR 8113 in December 1997, possibly for instrumental reasons, due to the rather low cadence of images, which missed the critical moment when the jet is seen. Another reason could be the choice of the coronal line of lower temperature in the case of TRACE observations (Fe IX and Fe X instead of Fe XII in case of EIT), closer to TR temperatures. The cadence of TRACE images was about $20 \mathrm{~s}$ and all temporal changes within the saddle structure were clearly visible.

Figure 4 shows changes in the magnetic field in AR 9636 from 1 to 4 October 2001. As seen in the left frame, it was a small active region with one single small sunspot of positive polarity surrounded by the network fields of the same polarity. The small patch of negative polarity near the NW edge of the sunspot likely arises due to the divergence of the positive magnetic flux and the location of the region not far from the West limb. On the next day a small bipolar region appears to the North of the sunspot. It becomes larger on 3 October, and its positive polarity is more developed. A fragment of negative polarity near the West border could also be due to the effect of projection. So, only one concentration of the negative polarity exists within the region occupied by the positive polarity. Obviously, a null point should appear above this concentration. Figure 5 shows the magnetic field level $-50 \mathrm{G}$ contours overlapped on the $171 \AA$ TRACE image. All images were aligned with the help of the full disk SOHO $195 \AA$ images obtained at the same time. It is evident that

1 Also called "magnetic field annihilation" in the Pikel'ner-Uchida (1969) analytic models for spicules. 


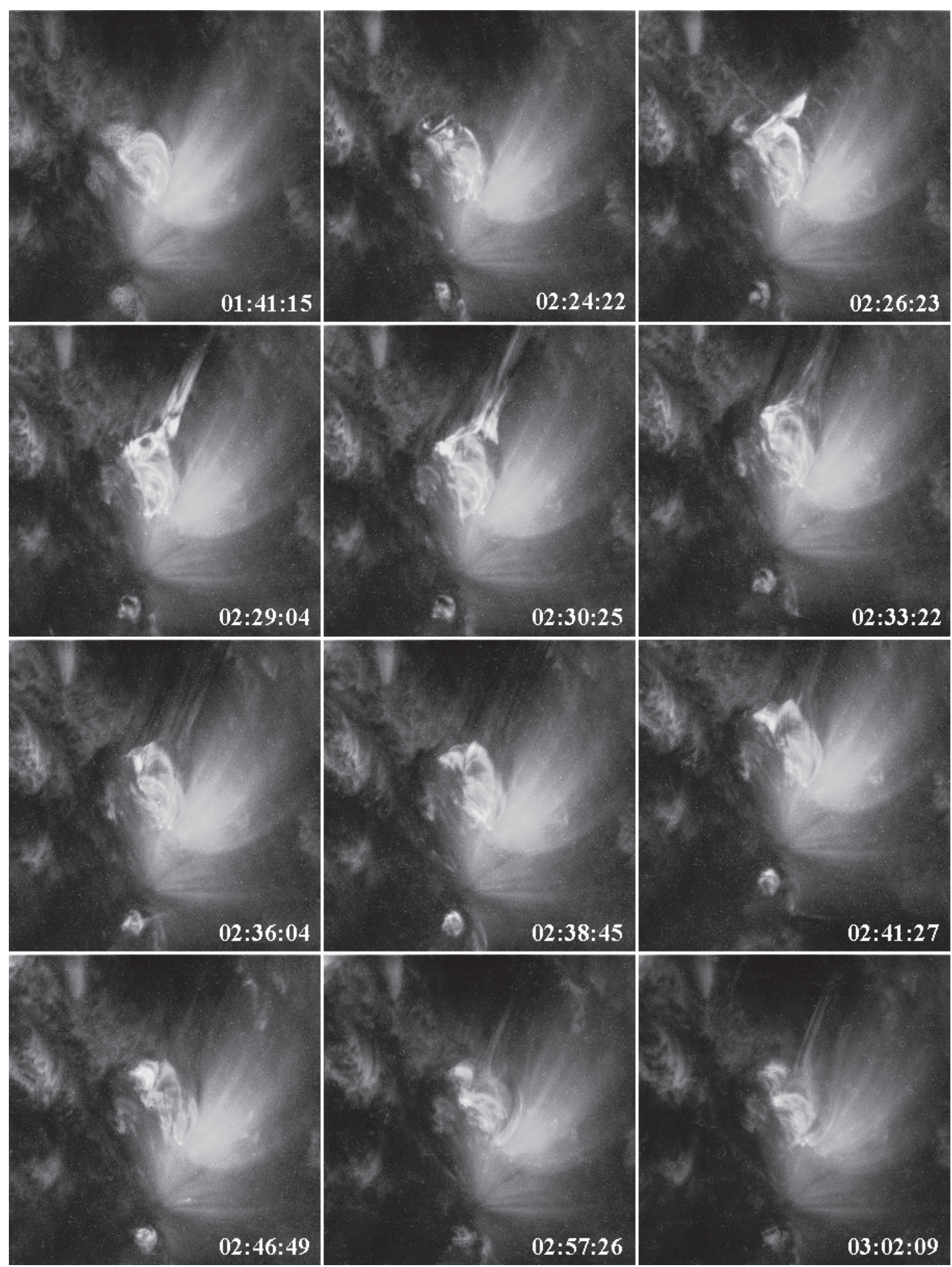

Fig. 3. Sequence of TRACE $171 \AA$ images showing a jet formation within a saddle structure on 3 October 2001. The size of each frame is about 2.3' $\times 2.3^{\prime}$. TRACE is a mission of the Stanford-Lockheed Institute for Space Research (a joint program of the Lockheed-Martin Advanced Technology Center's Solar and Astrophysics Laboratory and Stanford's Solar Observatories Group) and part of the NASA Small Explorer program.

the saddle structure is located right above the patch of parasitic polarity within the nearby unipolar region.

Figure 6 presents a sketch of the assumed 3D geometry of the magnetic field (similar to the popular representation already suggested in $2 \mathrm{D}$ and even $3 \mathrm{D}$, by several authors, see the review by Longcope 2005). Frozen-in plasma motion during the growth of the emerging bipole is shown, too. During its emergence, nearly vertical field lines of the unipolar region should be rearranged from the left side of the bipole to the right side. Some field lines pass through the reconnection in the Xpoint, while most of them are simply able to flow around the null point (fan reconnection), which is impossible to represent in 2D geometry (Priest \& Titov 1996). A difference image obtained by subtracting the image at 02:57:26 UT from the image 

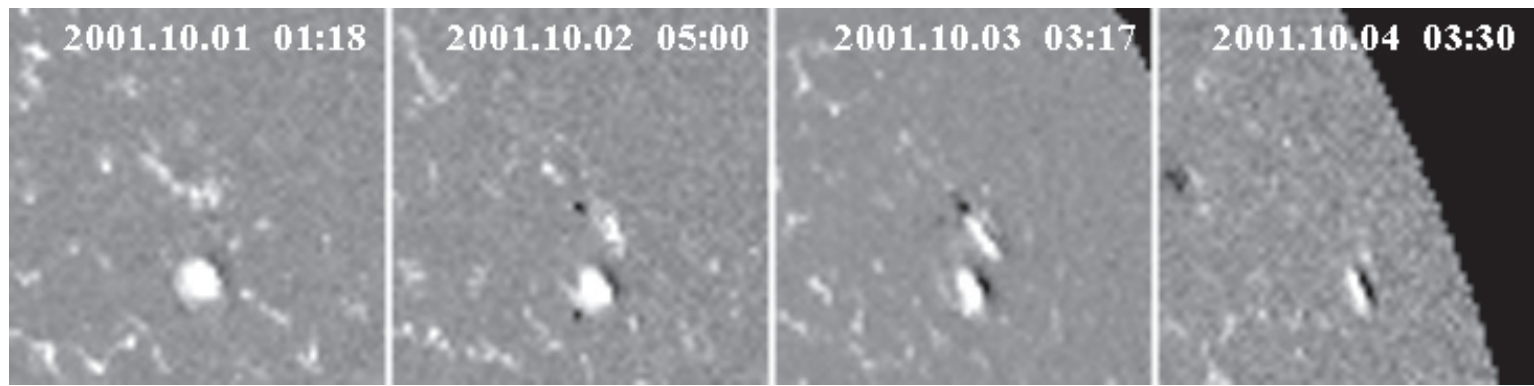

Fig. 4. Changes in the magnetic field of AR 9636 from 1 to 4 October 2001. Positive polarities are white and negative polarities are black. (Courtesy of SOHO/MDI consortium. SOHO is a project of international cooperation between ESA and NASA.)

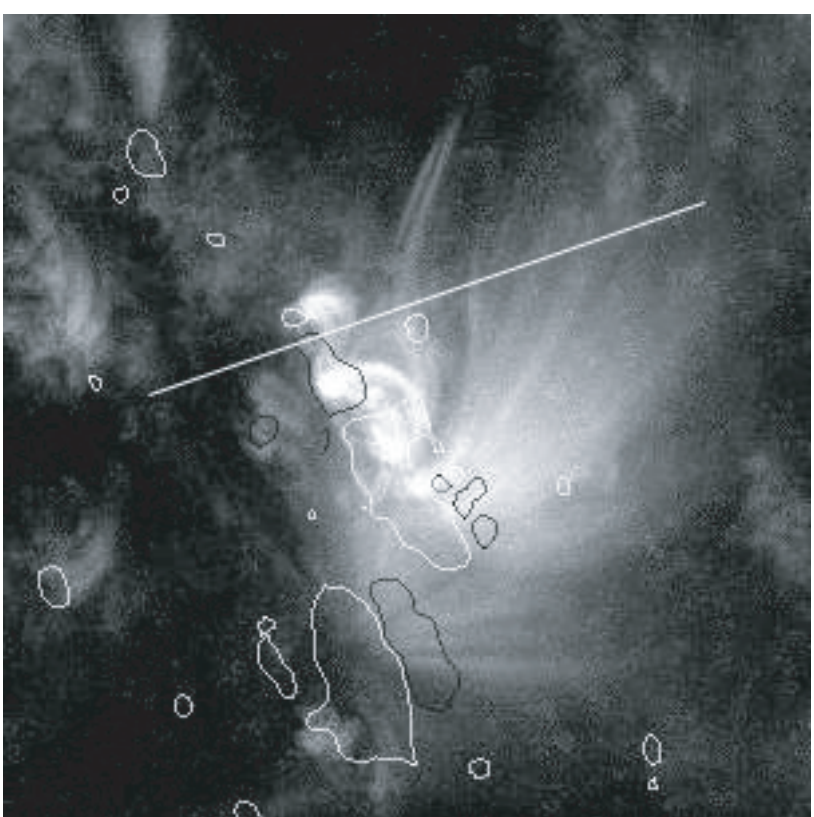

Fig. 5. Magnetic field level $\pm 50 \mathrm{G}$ contours overlapped on the $171 \AA$ TRACE image. Positive polarities are shown by white contours and negative polarities are shown by black contours. A straight line, drawn from the center of the disk, shows the projection of the local vertical direction.

at 03:04:10 UT (Fig. 7) shows the changes in the location of the coronal loops and the formation of new ones. Note in Fig. 7 that nearly straight fragments of loops to the right of the bipole are dual, consisting of a dark part at the left side of the structure and a bright part at the right side. These loops are rather stable and they show a displacement of flux tubes between 02:57:26 UT and 03:04:10 UT in agreement with the dashed lines in Fig. 6. Since both polarities of the emerging bipole are well developed, its magnetic moment is nearly horizontal. In contrast to a vertical bipole in a vertical external field, which forms a null point with a vertical spine curve, the horizontal bipole in a vertical field should form an X-point with a tilted spine curve. The tilt of the spine is clearly visible in Fig. 5 where the white straight line represents the local vertical direction.

In a $2 \mathrm{D}$ geometry, such a magnetic configuration was assumed in numerical modeling for producing plasma jets (Shibata et al. 1994; Yokoyama \& Shibata 1996). It is not evident that the same processes could happen in a $3 \mathrm{D}$ geometry with a single null point. Nevertheless, the distribution of the plasma observed in the UV shows that the process of reconnection really takes place in this region and the structure of the magnetic field is very close to what is expected for the vacuum magnetic field with the

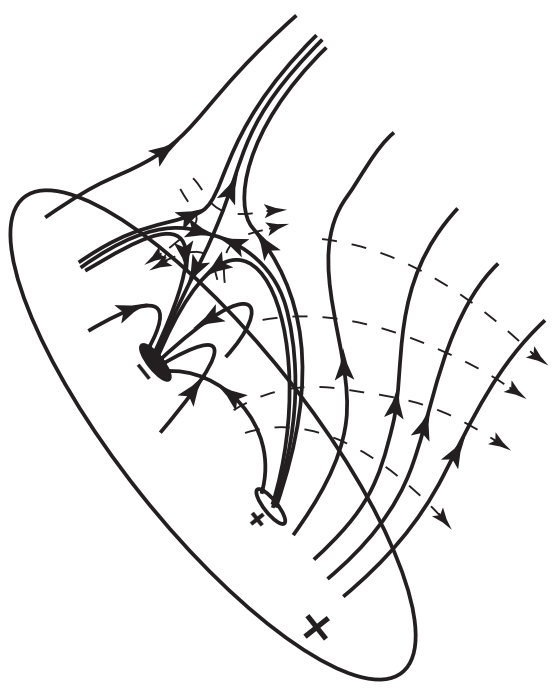

Fig. 6. A sketch of the 3D geometry of the magnetic field in the region of the jet formation. The dashed lines show the plasma displacement during the growth of the bipole.

same distribution of magnetic fields in the photosphere. Without considering the volume very close to the null point, the presence of a rare coronal plasma (with $\beta \ll 1$ ) cannot influence the magnetic field evolution. The frozen-in plasma motion will evolve in accordance with the expression

$\boldsymbol{v}_{\mathrm{d}}=c \frac{\boldsymbol{E} \times \boldsymbol{B}}{B^{2}}$

where

$\boldsymbol{E}=-\frac{1}{c} \frac{\partial \boldsymbol{A}}{\partial t}$

is the induction electric field, and $\boldsymbol{A}$ is the vector potential of the magnetic field $\boldsymbol{B}$. Transient inhomogeneities of the velocity field inevitably result in the formation of regions with plasma compression and rarefaction, in agreement with the equation of continuity

$\frac{\partial \rho}{\partial t}+\operatorname{div} \rho \boldsymbol{v}=0$

Plasma flows in the vicinity of a null point in a 2D geometry were studied by Somov \& Syrovatskii $(1971,1974)$ and more recently by Filippov (1997). Hyperbolically shaped field lines lead to plasma compression in two diametrically opposed quadrants, where flows converge (see Fig. 6), and rarefaction in two other quadrants, where flows diverge. The action of this geometrical factor is enhanced by the effect of the acceleration of plasma 


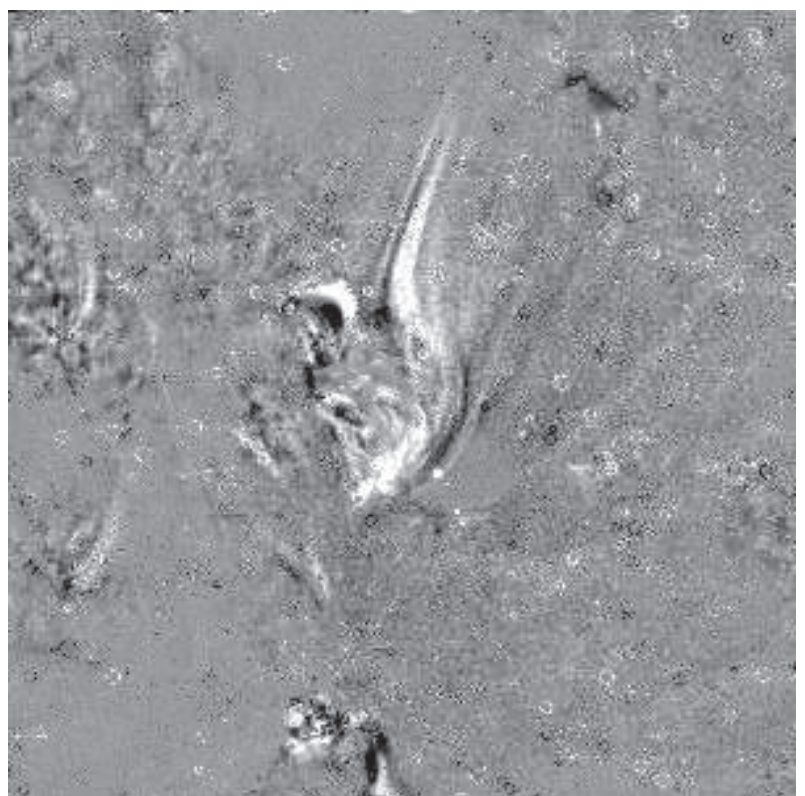

Fig. 7. TRACE difference image (03:04:10 UT - 02:57:26 UT) showing the displacement in coronal loops and the formation of new ones.

approaching the null point according to Eq. (1), as $B$ decreases (div $v>0$ ). Plasma that outflows from the null point decelerates (div $v<0$ ). So, regions of enhanced density (and enhanced pressure) should appear right-and-above the center of the saddle structure in the TRACE images and left-and-down of it during the growth of the bipole. We do indeed find two bright features in the difference image located just in these two quadrants, namely, a small arch to the left of the saddle in Fig. 7, both ends of which are anchored in the photosphere, and to the right of it, a jet-like structure along the spine.

Thus, field-aligned motion arises above the null point created in the corona by the emerging magnetic bipole leading to a "sudden" restructuring. The scale of the bipole is large enough to reveal the saddle structure around the $3 \mathrm{D}$ null point in the TRACE images; however, it is rather difficult to recognize it in lower resolution and "hotter" EIT images. The structure is clearly visible only in difference EIT images, in contrast to the case of the AR 8113 saddle structure, which can easily be found in EIT images described in Filippov (1999). For a smaller scale emerging magnetic bipole we would not be able to see a saddle structure created by its interaction with the background magnetic field, but would still observe the field-aligned jet-like motion. A lot of small jets could be created by very small magnetic elements that are found everywhere on the solar surface (Harvey \& Harvey 1973; Harvey \& Martin 1973; Harvey 1977; Title \& Schrijver 1998). These small jets could be identified as spicules, dark mottles, macrospicules, or surges. Their shapes and direction are defined by the geometry of the larger-scale ambient magnetic field and the orientation of the magnetic moment of emerging ephemeral magnetic regions. For example, the spine of the AR 8113 null point was nearly a vertical line, while the spine in AR 9636 was tilted to the vertical direction by about $45^{\circ}$. A host of jet-like structures (jetlets) forms the dynamic upper chromospheric shell that shows the prolateness at the epoch of low activity. Although it is still difficult to observe the null points and the small-scale saddle structures within the low chromosphere (see Fig. 1) we believe that the physics is the same as in the processes in the larger-scale saddle structures in the corona where plasma motion is visible more clearly. Inside the dynamical chromospheric shell, the proposed corresponding magnetic structure is much smaller in size, which makes it difficult to perform observations in greater detail; accordingly, the application of the model proposed from the "solid" TRACE observations to the numerous spicule events of the chromosphere is still speculative. Hopefully, the new observations planned in space with Solar-B and SDO will permit a resolution of this issue.

\section{Conclusions}

We analyzed a jet-like event observed by TRACE on 3 October 2001 above a structure that could be recognized as a saddle structure around a null (X-type) point. This null point was created by an emerging nearly horizontal bipole and the ambient vertical magnetic field. The growth of the bipole leads to a reconnection of the field lines and to a specific plasma motion in the vicinity of the null point that results in a plasma flow along the spine line of the 3D null. We believe that similar but smaller processes could happen very often at a smaller scale in the chromosphere, near emerging magnetic ephemeral regions, producing numerous jet-like structures (jetlets) in the upper chromosphere such as spicules, dark mottles, giant spicules, macrospicules, spikes, and surges, as proposed by Yamauchi et al. (2005). Assuming that this structure is "universal" and exists on a very small scale (see Fig. 1), the geometry of the ambient magnetic field influences the jet trajectories and should determine the average density distribution in the upper chromosphere. At the epoch of low activity, the difference in the largescale structure of the polar magnetic field and that of the low latitude quiet region magnetic field results in a prolateness of the chromosphere (Auchere et al. 1998; Zhang et al. 1980; Filippov \& Koutchmy 2000; Vilinga \& Koutchmy 2005).

It is however not yet clear why we do not see a radiative signature of the reconnections in the heart of the chromosphere: where is the site of the accelerated particles responsible for the jet-like phenomenon? Other related questions include why are macro-spicules produced mainly in coronal holes and not in other locations? Is there a connection between the conditions leading to macro-spicules and the observed greater extent of the chromosphere? Is the seemingly lifting effect related to the rate of emergence of small magnetic elements? New more systematic observations of both the solar-cycle behavior of the chromospheric prolateness, including the study of spicules on the disk and the changes of the corresponding photospheric magnetic structures, and the detailed observations of macro-spicules, including their SXR counterpart (Koutchmy et al. 1998) and their possible cool counterpart, are needed. The forthcoming new space missions will offer an excellent opportunity to address these questions. Ground-based routine observations of the shape of the chromospheric shell and microwaves observations (1 to $8 \mathrm{~mm}$ wavelengths and further) of polar regions and polar cap brightenings (see, for example, Efanov et al. 1980; Debeve et al. 1979; Shibasaki 1998) can also provide some interesting diagnostics.

Acknowledgements. We thank L. Golub for reading the manuscript of this paper and for making meaningful suggestions for improving it. This work was supported in part by the Russian Foundation for Basic Research (grant 06-02-16424) and in part by the NATO CRG 940291.

\section{References}

Athay, G. 1959, ApJ, 129, 164

Athay, G. 1986, in Physics of the Sun, ed. P. A. Sturrock, Vol. II (Reidel Pub.) 51 
Auchere, F., Boulade, S., Koutchmy, S., et al. 1998, A\&A, 366, L57

Beckers, J. M. 1972, ARA\&A, 10, 73

Belenko, I. A. 2001, Sol. Phys., 199, 23

Blake, M. L., \& Sturrock, P. A. 1985, ApJ, 290, 359

Chae, J., Qiu, J., Wang, H., \& Goode, P. R. 1999, ApJ, 513, L75

Christopoulou, E. B., Georgakilas, A. A., \& Koutchmy, S. 2001, Sol. Phys., 199, 61

Daw, A., DeLuca, E. E., \& Golub, L. 1995, ApJ, 453, 929

Debeve, G., Koutchmy, S., Efanov, V. A., \& Moisseev, I. G. 1979, J. Opt. (Paris), 10,339

Dunn, R. B. 1960, Ph.D. Harvard Univ. Mass., 172, 23

Efanov, V. A., Moisseev, I. G., Nesterov, N. S., \& Steward, R. 1980, Izv. Krymsk. Astrofiz. Obs., 61, 52

Filippov, B. P. 1993, Soviet Ast., 37, 547

Filippov, B. P. 1997, A\&A, 324, 324

Filippov, B. P. 1999, Sol. Phys., 185, 297

Filippov, B., \& Koutchmy, S. 2000, Sol. Phys., 196, 311

Filippov, B. P., Koutchmy, S., \& Den, O. G. 2002, Il Nuovo Cimento, 25, 667

Fracastoro, M. G. 1948, Pub. R. Oss. Arcetri, 64, 44

Georgakilas, A. A., Koutchmy, S., \& Alissandrakis C. E. 1999, A\&A, 341, 610

Georgakilas, A. A., Koutchmy, S., \& Christopoulou, E. B. 2001, A\&A, 341, 610

Harvey, J. W. 1977, Highlights of Astronomy, 4, 223

Harvey, K., \& Harvey, J. 1973, Sol. Phys., 28, 61

Harvey, K. L., \& Martin, S. F., 1973, Sol. Phys., 32, 389

Henoux J. C., \& Somov, B. V., 1997, A\&A, 318, 947

Johannesson, A., \& Zirin, H. 1996, ApJ, 471, 510

Karpen, J. T., Antiochos, S. K., \& DeVore, C. R. 1995, ApJ, 450, 422

Karpen, J. T., Antiochos, S. K., DeVore, C. R., \& Golub, L. 1998, ApJ, 495, 491

Koutchmy, S., \& Loucif, M. L. 1991, in Mechanisms of Chromospheric and Coronal Heating, ed. P. Ulmschneider, E. R. Priest, \& R. Rosner (Berlin: Springer-Verlag) 152

Koutchmy, S., Hara, H., Suematsu, Y., \& Reardon, K. 1997, A\&A, 320, L33

Koutchmy, S., Hara, H., Shibata, K., Suematsu, Y., \& Reardon, K. 1998 in Astrophysics and Space Science Library 229, Observational Plasma Astrophysics: Five Years of Yohkoh and Beyond, ed. T. Watanabe, T. Kosugi, \& A. C. Sterling (Boston, Mass.: Kluwer Academic Publishers), 87
Lorrain, P., \& Koutchmy, S. 1996, Sol. Phys., 165, 115

Longcope, D. W. 2005, Living Rev. Sol. Phys., 2, 7

Pikel'ner, S. B. 1969, Soviet Ast., 13, 259

Platov, Yu. V., Somov, B. V., \& Syrovatskii, S. I. 1973, Sol. Phys., 30, 139

Priest, E. R., \& Titov, V. S. 1996, Phil. Trans. Roy. Soc. Lond. A., 354, 2951

Proceedings of the Guadeloupe Conference 1998, Solar Jets and Coronal Plumes, ESA SP-421

Roberts, W. O. 1946, ApJ, 101, 136

Rompolt, B., \& Svestka, Z. 1996, Adv. Space Res., 17, 115

Shibasaki, K. 1998, in Synoptic Solar Physics, ed. K. S. Balasubramanian, J. Harvey, \& D. Rabin, ASP Conf. Ser., 140, 373

Schluter, A. 1957, in Radio Astronomy, ed. H. C. van de Hulst (Cambridge Univ. Press), IAU Symp., 4, 356

Secchi, S. J. 1877, in Le Soleil (Gauthier-Villars) 38

Shibata, K., \& Uchida, Y. 1986, Sol. Phys., 103, 299

Shibata, K., Ishido, Y., Acton, L. W., et al., 1992, PASJ, 44, L173

Shibata, K., Nitta, N., Matsumoto, R., et al. 1994, in X-ray Solar Physics from Yohkoh, Proc. of the International Symp. on the Yohkoh Scientific Results, ed. Y. Uchida, T. Watanabe, K. Shibata, \& H. S. Hudson (Tokyo: Universal Academy Press), 29

Somov, B. V., \& Syrovatskii, S. I. 1971, JETF, 61, 621.

Somov, B. V., \& Syrovatskii, S. I. 1974, in Trudy FIAN 74, Neitralnye Tokovye Sloi v Plazme (Moscow: Nauka), 14

Sterling, A. C. 2000, Sol. Phys., 196, 79

Title, A. M., \& Schrijver, C. J. 1998, in Cool Stars, Stellar Systems and the Sun, ed. R. A. Donahue, \& J. A. Bookbinder, ASP Conf. Ser., 154, 345

Uchida, Y. 1969, PASJ, 21, 128

Varsik, J. R., Wilson, P. R., \& Li, Y. 1999, Sol. Phys., 184, 223

Vilinga, J., \& Koutchmy, S. 2005, in Large-Scale Structures and Their Role in Solar Activity, Proc. of the 22d NSO-SPD Workshop, ed. K. Sankarasubramanian, M. Penn, \& A. Pevtsov, ASP Conf. Ser., 346, 269

Wang, Y.-M., Sheeley, N. R., Jr., Socker, D. G., et al. 1998, ApJ, 508, 899

Yamauchi, Y., Wang, H., Jiang, Y., Schwandron, N., \& Moore, R. L. 2005, ApJ, 629,572

Yokoyama, T., \& Shibata, K. 1996, PASJ, 48, 353

Zhang, J., White, S. M., \& Kundu, M. R. 1998, ApJ, 504, L127 